\title{
Factores de riesgo asociados al control glucémico y síndrome metabólico en pacientes con diabetes mellitus tipo 2. Villavicencio, Colombia
}

\author{
Risk factors associated with glycemic control and metabolic syndrome in patients with type 2 diabetes mellitus. \\ Villavicencio, Colombia
}

Flor Stella Piñeros-Garzón 1* orcid.org/0000-0002-8824-2041

Jorge Martín Rodríguez-Hernández² orcid.org/0000-0002-7301-7706

1 Univ ersidad de Los Llanos. Villav icencio, Colombia

2 Instituto de Salud Pública, Pontificia Univ ersidad Jav eriana. Bogotá, Colombia

Fecha de recepción: Enero 21 - $2018 \quad$ Fecha de revisión: Nov iembre 10-2018 Fecha de aceptación: Diciembre 11 - 2018

Piñeros-Garzón FS, Rodríguez-Hernández JM. Factores de riesgo asociados al control glucémico y síndrome metabólico en pacientes con diabetes mellitus tipo 2. Villavicencio, Colombia. Univ. Salud. 2019;21(1):61-71. DOI: http://dx.doi.org/10.22267/rus.192101.140

\section{Resumen}

Introducción: La Diabetes Mellitus tipo 2 (DMT2) es una enfermedad crónica cuya prevalencia elevada hace que sea un importante problema de salud pública, social y económico en los países. Objetivo: Identificar factores de riesgo potencialmente asociados al control glucémico y Síndrome Metabólico (SM) de pacientes con DMT2 de una Institución prestadora de Servicios de Salud (IPS) de Villavicencio. Materiales y métodos: Estudio analítico transversal a partir de información secundaria. Se establecieron modelos multivariados basados en regresiones binomiales para analizar razones de prevalencia ajustadas en dos variables: síndrome metabólico y control glucémico. Resultados: Más del 90\% de los individuos eran mayores de 49 años; 50,6\% hombres; 46,6\% presentó cifras de la prueba de hemoglobina glicosilada (HbA1c) mayor a 7\%; 64,5\% tenían SM. Los individuos con hiperglicemia tuvieron 3,1 veces más riesgo de tener inadecuado control glucémico (IC 95\%:2,28-4,25, p<0,05); aquellos con hipotiroidismo presentaron 1,2 veces más riesgo de desarrollar SM (IC 95\%:1,01-1,35; $\mathrm{p}<0,05$ ) y aquellos con cardiopatía tuvieron 1,3 veces más riesgo de desarrollar SM. Conclusiones: A pesar de ser una población controlada y en proceso de monitoreo, alto porcentaje de pacientes presentó inadecuado control metabólico, aumentando el riesgo cardiovascular, esto sugiere optimizar procesos de seguimiento institucional.

Palabras clave: Diabetes mellitus tipo 2; síndrome metabólico; hemoglobina A glucada. (Fuente: DeCS, Bireme).

\begin{abstract}
Introduction: Type 2 diabetes mellitus (T2DM) is a chronic disease whose high prevalence makes it an important public, social and economic health problem in countries. Objective: To identify risk factors potentially associated with glycemic control and metabolic syndrome (MS) of patients with T2DM of a Health Services Institution (IPS) in Villavicencio, Colombia. Materials and methods: A transverse analytical study from secondary information was made. Multivariate models based on binomial regressions were established to analyze reasons of prevalence adjusted in two variables: metabolic syndrome and glycemic control. Results: More than $90 \%$ of individuals were over 49 years old; $50.6 \%$ were men; $46.6 \%$ showed the glycosylated hemoglobin test (HbA1c) figures greater than $7 \%$; $64.5 \%$ had MS. Individuals with hyperglycemia had 3.1 times the risk of inadequate glycemic control $(95 \%$ CI: 2,28-4,25, p < 0.05); Those with hypothyroidism presented 1.2 times more risk of developing MS (95\% CI: 1,01-1,35; $\mathrm{p}<0.05)$ and those with heart disease had 1.3 times the risk of developing MS. Conclusions: Despite being a controlled population and in the process of monitoring, high percentage of patients presented inadequate metabolic control which increases cardiovascular risk. This suggests optimizing institutional follow-up processes.
\end{abstract}

Key words: Diabetes mellitus, type 2; metabolic syndrome; glycated hemoglobin A. (Source: DeCS, Bireme).

*Autor de correspondencia

Flor Stella Piñeros Garzón

e-mail: fpineros@unillanos.edu.co 


\section{Introducción}

La diabetes mellitus (DM) es una enfermedad crónica que según el informe mundial de la Organización Mundial de la Salud (OMS) ${ }^{(1)}$ ha presentado incremento en su prevalencia, en especial en países de bajos y medianos ingresos; su ocurrencia es considerada por la OMS y la Federación Internacional de Diabetes (FID) como una " epidemia mundial" que afecta las personas en su edad más productiva, empobreciendo a las familias y disminuyendo la esperanza de vida, convirtiéndose así en una de las enfermedades con mayor impacto socio sanitario. Junto con las otras tres principales enfermedades no transmisibles (ENT) (cardiovasculares, cáncer y enfermedades respiratorias), representa más del $80 \%$ de todas las muertes prematuras por estas causas(2). La prevalencia de esta enfermedad a nivel mundial en mayores de 18 años ha aumentado del 4,7\% (1980) al 8,5\% (2014)(1); en Colombia la prevalencia de diabetes mellitus tipo 2 (DMT2) oscila entre $4 \%$ al $8 \%(3)$.

Según el Plan de Acción Mundial de la OMS para la prevención y el control de las ENT(4), se ha propuesto para el 2020 una reducción relativa del $25 \%$ en la mortalidad prematura por enfermedades cardiovasculares, en las que se encuentra la DM, para lo cual el principal objetivo es un adecuado control glucémico. La Asociación Americana de Diabetes (ADA) y la Asociación Latinoamericana de Diabetes (ALAD)(5) han establecido como meta de control un nivel de hemoglobina glicosilada $\mathrm{HbA1c} \leq 7 \%$, aunque sugiere que este objetivo puede reducirse más en pacientes de reciente diagnóstico sin complicaciones conocidas, con larga esperanza de vida, y ser menos estrictos en pacientes con complicaciones vasculares muy evolucionadas y en situaciones en las que el recambio de eritrocitos está aumentado, como en la anemia hemolítica e insuficiencia renal y en estados que aumentan la formación de eritrocitos, como ocurre posterior a la administración de hierro(6,7); estas referencias son tomadas en Colombia mediante la Guía de Práctica Clínica (GPC) para el diagnóstico, tratamiento y seguimiento de la diabetes mellitus tipo 2 en la población mayor de 18 años.
Con relación a los valores de glucemia, la ALAD ha establecido como meta, en ayuno valores entre 70 a $120 \mathrm{mg} / \mathrm{dl}$.

Otros aspectos importantes que se asocian frecuentemente con la condición de DM, son la obesidad, alteraciones del metabolismo lipídico y proteínico, así como con hipertensión arterial (HTA) y otros factores de riesgo cardiovascular, que conllevan a una situación denominada síndrome metabólico (SM)(8). Existen varias definiciones, pero por basarse en criterios clínicos y pruebas de laboratorio sencillas, el más utilizado en la población general es el del Programa Nacional de Educación sobre el Colesterol (NCEP por sus siglas en inglés) y el Panel III de Tratamiento del Adulto (ATP III) que define SM cuando se cumplen tres o más de los siguientes criterios: glicemia $\geq 100 \mathrm{mg} / \mathrm{dl} \quad \mathrm{o}$ antecedente de DM, circunferencia de cintura en hombres $\geq$ a $102 \mathrm{~cm}$, en mujeres $\geq$ a $88 \mathrm{~cm}$, tensión arterial $\geq 130 / 85 \mathrm{mmHg}$ en ambos sexos, lipoproteínas de alta densidad o c-HDL en hombres menor a $40 \mathrm{mg} / \mathrm{dl}$ y en mujeres menor a $50 \mathrm{mg} / \mathrm{dl}$, y valor de triglicéridos $\geq 150 \mathrm{mg} / \mathrm{dl}$ en ambos sexos( ${ }^{(9)}$.

Para valorar el daño renal, usualmente se tiene en cuenta la clasificación de la Enfermedad Renal Crónica (ERC), según tasa de filtración glomerular (TFG) método rápido y confiable reconocido mundialmente para estimar ERC según estadios en pacientes con DMT2(10). De acuerdo al análisis de glutamato-piruvato transaminasa (GPT) en Colombia, la TFG se calcula utilizando fórmulas como MDRD, Cockroft o CKD-Epi que tienen en cuenta la creatinina sérica, la edad, en algunas el peso y se ajustan por sexo (mujer) y por raza negra(11).

En Colombia, mediante el Acuerdo 117 de 1998, se incluyó la DM como una de las enfermedades de interés en salud pública que debe ser objeto de atención oportuna y seguimiento para garantizar su control y reducir complicaciones evitables. Actualmente, todas las personas con DM deben estar inscritas en el Programa de Riesgo Cardiovascular (PRCV), que ha sido una de las principales estrategias del Ministerio de Salud para contribuir a reducir la 
morbimortalidad asociada a eventos cerebrovasculares (ECV), cuyo principal propósito es incorporar el enfoque de riesgo cardiovascular (CV) global en el manejo de las personas bajo control. Por su parte dentro del Plan Decenal de Salud Pública (PDSP) 2012 2021 se tiene como objetivo reducir la mortalidad prematura por DM en $25 \%$ en población entre 30 a 70 años(12), mediante la implementación de las Rutas Integrales de Atención en Salud (RIAS), que definen en Colombia las condiciones necesarias para asegurar la integralidad en la atención.

El Meta, es uno de los departamentos con prevalencia más elevada de esta patología con respecto a la referencia nacional, según el último Análisis de Situación en Salud del departamento, para 2013 la tasa de mortalidad ajustada por edad de DM fue dos veces mayor al indicador nacional $(16,43 \%)$ siendo 31,1 por cada 100.000 habitantes(13). En el caso de Villavicencio, la DM desde 2010 ha sido el evento que aporta la mayor mortalidad por año, presentando para 2013 una tasa de 39,8 por cada 100.000 habitantes(14). Por lo anterior, y dada la importancia de controlar clínicamente esta población, donde cada vez la prevalencia de DM supera la media nacional y no se cuenta con información sobre las características de usuarios que comúnmente deben estar inscritos en un programa de seguimiento, se realizó este estudio, que busca identificar factores de riesgo potencialmente asociados al control glucémico y síndrome metabólico de pacientes con DMT2 de una Institución prestadora de Servicios de Salud (IPS) de Villavicencio, en 2016, a partir de variables sociodemográficas y clínicas, valoradas comúnmente dentro del Programa de seguimiento.

\section{Materiales y métodos}

Se realizó un estudio analítico de corte transversal para analizar información de pacientes con DMT2, a partir de información sociodemográfica y clínica extraída de registros de controles periódicos de un grupo de pacientes que acudieron al PRCV de una IPS en el año 2016.
Los datos se recolectaron en el segundo trimestre de 2017.

El universo fueron las personas con DMT2, que se encontraban inscritas y activas en el programa de riesgo cardiovascular de la IPS privada de bajo nivel de complejidad. Se incluyeron los pacientes que asistieron a la consulta de control desde enero de 2016 a diciembre del mismo año, siendo 539 los individuos encontrados en la base de seguimiento, de los cuales 453 fueron seleccionados para el estudio, según criterios de inclusión.

Los sujetos se seleccionaron a partir del censo de personas con DMT2, activas del Programa descrito. Los criterios de inclusión en el estudio fueron: usuarios activos en el PRCV de la IPS con corte a diciembre de 2016 y que tuvieran registros en base de datos de seguimiento a pacientes crónicos, valoración de control por parte del programa evidenciado por historia clínica de seguimiento y reporte de perfil bioquímico de los últimos 12 meses. Para el logro de los objetivos, se estudiaron variables sociodemográficas y clínicas.

Las fuentes de información correspondieron a los registros de datos secundarios de los usuarios almacenados en formatos de monitoreo y seguimiento, complementados con la revisión de la historia clínica de control y el software con los registros de los resultados del perfil bioquímico de los usuarios de la IPS.

Variables independientes

- Sociodemográficas: edad, sexo, estado civil y zona de residencia

- Clínicas: peso, índice de masa corporal, niveles séricos de triglicéridos, colesterol HDL, colesterol LDL, colesterol total, HbA1c, glucemia en ayunas, creatinina, reporte de presión arterial sistólica y presión arterial diastólica, tasa de filtración glomerular (determinada por formula de CockcroftGault), factores de riesgo (hábito de fumar, alcoholismo, dislipidemia), comorbilidades y complicaciones. 
Variables dependientes

- Control glucémico, considerado como control, sujetos con valores de HbA1c $\leq 7 \%$ y no control, valores mayores $(5,6)$

- Síndrome Metabólico, según Tercer Panel de Tratamiento del Adulto (ATP III) ${ }^{(9)}$ teniendo en cuenta que la variable perímetro abdominal no fue incluida porque presentó subregistro en la muestra analizada, los criterios fueron: valores de triglicéridos $\leq$ $150 \mathrm{mg} / \mathrm{dl}$; colesterol HDL < $50 \mathrm{mg} / \mathrm{dl}$ en mujeres $\mathrm{y}<40 \mathrm{mg} / \mathrm{dl}$ en hombres, cifras tensionales $\geq 130 / 85 \mathrm{mmHg}$ o antecedente personal de hipertensión arterial. Se consideró que el individuo presentaba dicha condición si tenía 2 o más de estos criterios puesto que partían del hecho de ser diabéticos.

Para el análisis de datos se calcularon frecuencias y porcentajes para las variables categóricas. Se usó la prueba de Chi cuadrado para comparar la proporción de usuarios según cada una de las variables dependientes, por las diferentes categorías de variables independientes, y así se determinó la existencia de diferencias significativas con un valor $\mathrm{p}<0,05$.

Posteriormente, se establecieron Razones de Prevalencia (RP) crudas con sus respectivos intervalos de confianza al 95\% (IC 95\%) y nivel de significancia menor a 0,05 para todas las variables independientes ( 0 y 1$)$, clasificando como "0" la categoría "bajo riesgo o ausencia de exposición" y "1" la categoría correspondiente a "mayor riesgo o presencia de exposición" según evidencia científica.

Finalmente, se realizó un análisis múltiple, mediante el modelo de regresión binomial controlado por las variables potencialmente confusoras para obtener RP ajustadas con IC $95 \%$ y nivel de significancia $<0,05$; las variables ingresadas en este modelo fueron aquellas que en el análisis bivariado presentaron valores de p $<0,25$ (según Hosmer Lemeshow) o que según literatura mostraran relación con la variable dependiente. El procesamiento de la base de datos y los análisis estadísticos fueron realizados con Stata versión 12(15) y SPSS versión 22(16).

\section{Consideraciones éticas}

De acuerdo con la normatividad colombiana establecida en la Resolución 8430 de 1993 del Ministerio de Salud, el presente estudio es clasificado como investigación "sin riesgo". Se cumplieron las recomendaciones de la declaración de Helsinki, debido a que la información obtenida de fuentes secundarias se usó de forma confidencial desde la IPS quien custodia los datos de sus usuarios y autorizó el acceso a la información. Posterior a verificar en las diferentes fuentes de información los datos faltantes, la base fue anonimizada. El protocolo de investigación fue aprobado para su realización por el Comité de Ética de la Universidad de los Llanos.

\section{Resultados}

En el estudio fueron incluidos 453 sujetos, 50,6\% hombres, de éstos $91,3 \%$ y $93,8 \%$ de las mujeres tenía 50 años o más. En estado civil, se consideraron 394 sujetos, puesto que el restante no permitía definir si tenía o no pareja, predominaron individuos casados y en unión libre. El 3,9\% de hombres y 3,1\% de mujeres habitaban en zona rural. No hubo diferencia en las características sociodemográficas estudiadas según sexo $(p>0,05)$ (Tabla 1$)$.

Con respecto a las características clínicas, los valores de glucemia en ayunas que predominaron correspondieron a hiperglucemia (> $130 \mathrm{mg} / \mathrm{dl}$ ), estando presente en $65,4 \%$ de hombres y $57,0 \%$ de mujeres $(\mathrm{p}=0,067)$. En lo relacionado al perfil lipídico, 69,0\% de hombres y $56,2 \%$ de mujeres presentaron hipercolesterolemia (colesterol total > 200 $\mathrm{mg} / \mathrm{dl}$ ); 53,7 \% de los hombres y 63,8\% de las mujeres presentaron valores de c-HDL inferiores; $49,8 \%$ de los hombres y $58,9 \%$ de las mujeres presentaron niveles altos de c-LDL (>100 mg/dl), estas características mostraron diferencia por sexo; los niveles anormales de triglicéridos o hipertrigliceridemia $\quad \geq 150$ $\mathrm{mg} / \mathrm{dl}$ ) no evidenciaron diferencias por sexo $(p=0,548)$. Datos no mostrados en las tablas. 
Tabla 1. Características sociodemográficas y clínicas según control glucémico de personas con DMT2

\begin{tabular}{|c|c|c|c|c|c|}
\hline \multirow[b]{2}{*}{ Variable } & \multirow[b]{2}{*}{$\mathbf{n}$} & \multicolumn{2}{|c|}{ Control glucémico (HbA1c) } & \multirow[b]{2}{*}{$\begin{array}{c}\text { RP cruda } \\
\text { IC } 95 \%\end{array}$} & \multirow[b]{2}{*}{ Valor $\mathbf{p}$} \\
\hline & & $\begin{array}{c}\text { Control } \\
\text { (Hba1c } \leq \mathbf{7 \%})[242] 53,4 \% \\
\end{array}$ & $\begin{array}{c}\text { No control } \\
\text { (Hba1c }>\mathbf{7 \%} \text { ) }[211] 46,6 \% \\
\end{array}$ & & \\
\hline \multicolumn{6}{|l|}{ Sociodemográficas } \\
\hline \multicolumn{6}{|l|}{ Edad } \\
\hline$<50$ años & 34 & $19\{7,9\}$ & $15\{7,1\}$ & Referencia & 0,770 \\
\hline$\geq 50$ años & 419 & $223\{92,1\}$ & $196\{92,9\}$ & $1,06(0,72-1,57)$ & \\
\hline \multicolumn{6}{|l|}{ Sexo } \\
\hline Hombre & 229 & $110\{45,5\}$ & $119\{56,4\}$ & Referencia & 0,021 \\
\hline Mujer & 224 & $132\{54,5\}$ & $92\{43,6\}$ & $0,79(0,65-0,97)$ & \\
\hline \multicolumn{6}{|l|}{ Zona de residencia } \\
\hline Urbana & 437 & $232\{95,9\}$ & $205\{97,2\}$ & Referencia & 0,493 \\
\hline Rural & 16 & $10\{4,1\}$ & $6\{2,8\}$ & $0,80(0,42-1,52)$ & \\
\hline \multicolumn{6}{|l|}{ Estado Civil a } \\
\hline Con pareja & 244 & $127\{60,2\}$ & $117\{63,9\}$ & Referencia & 0,450 \\
\hline Sin pareja & 150 & $84\{39,8\}$ & $66\{36,1\}$ & $0,92(0,73-1,15)$ & \\
\hline \multicolumn{6}{|l|}{ Clínicas } \\
\hline \multicolumn{6}{|c|}{ Síndrome metabólico } \\
\hline No & 161 & $89\{36,8\}$ & $72\{34,1\}$ & Referencia & 0,559 \\
\hline $\mathrm{Si}$ & 292 & $153\{63,2\}$ & $139\{65,9\}$ & $1,06(0,86-1,31)$ & \\
\hline \multicolumn{6}{|c|}{ Cifras TA en consulta } \\
\hline$<130 / 85 \mathrm{mmHg}$ & 400 & $209\{86,4\}$ & $191\{90,5\}$ & Referencia & 0,201 \\
\hline$\geq 130 / 85 \mathrm{mmHg}$ & 53 & $33\{13,6\}$ & $20\{9,5\}$ & $0,79(0,55-1,13)$ & \\
\hline \multicolumn{6}{|c|}{ Glicemia en ayunas ${ }^{a}$ b } \\
\hline 70 a 120 mg/dl & 175 & $140\{58,1\}$ & $35\{16,7\}$ & Referencia & $<0,001$ \\
\hline$>120 \mathrm{mg} / \mathrm{dl}$ & 276 & $101\{41,9\}$ & $175\{83,3\}$ & $3,17(2,33-4,32)$ & \\
\hline \multicolumn{6}{|l|}{ Obesidad (IMC $\geq 30$} \\
\hline $\mathrm{Kg} / \mathrm{m}^{2)}$ & 287 & $151\{62,4\}$ & $136\{64,5\}$ & Referencia & \\
\hline No & 166 & $91\{37,6\}$ & $75\{35,5\}$ & $0,95(0,78-1,17)$ & 0,652 \\
\hline $\mathrm{Si}$ & & & & & \\
\hline \multicolumn{6}{|l|}{ ERC $^{c}$} \\
\hline Estadios I a III & 443 & $238\{98,3\}$ & $205\{97,2\}$ & Referencia & 0,324 \\
\hline Estadios IV y V & 10 & $4\{1,7\}$ & $6\{2,8\}$ & $1,30(0,77-2,17)$ & \\
\hline \multicolumn{6}{|l|}{ Dislipidemia $^{\mathrm{d}}$} \\
\hline No & 112 & $59\{24,4\}$ & $53\{25,1\}$ & Referencia & 0,855 \\
\hline $\mathrm{Si}$ & 341 & $183\{75,6\}$ & $158\{74,9\}$ & $0,98(0,78-1,23)$ & \\
\hline \multicolumn{6}{|c|}{ Antecedente de HTA } \\
\hline No & 170 & $85\{35,1\}$ & $85\{40,3\}$ & Referencia & 0,253 \\
\hline $\mathrm{Si}$ & 283 & $157\{64,9\}$ & $126\{59,7\}$ & $0,89(0,73-1,09)$ & \\
\hline \multicolumn{6}{|l|}{ Hiperuricemia } \\
\hline No & 436 & $231\{95,5\}$ & $205\{97,2\}$ & Referencia & 0,388 \\
\hline $\mathrm{Si}$ & 17 & $11\{4,5\}$ & $6\{2,8\}$ & $0,75(0,39-1,44)$ & \\
\hline \multicolumn{6}{|l|}{ Hipotiroidismo } \\
\hline No & 358 & $193\{79,8\}$ & $165\{78,2\}$ & Referencia & 0,682 \\
\hline $\mathrm{Si}$ & 95 & $49\{20,2\}$ & $46\{21,8\}$ & $1,05(0,83-1,33)$ & \\
\hline \multicolumn{6}{|c|}{ Enfermedad cardiaca } \\
\hline No & 417 & $220\{90,9\}$ & $197\{93,4\}$ & Referencia & 0,366 \\
\hline $\mathrm{Si}$ & 36 & $22\{9,1\}$ & $14\{6,6\}$ & $0,82(0,54-1,26)$ & \\
\hline Nefropatía & & & & & \\
\hline No & 421 & $224\{92,6\}$ & $197\{93,4\}$ & Referencia & 0,745 \\
\hline $\mathrm{Si}$ & 32 & $18\{7,4\}$ & $14\{6,6\}$ & $0,93(0,62-1,40)$ & \\
\hline
\end{tabular}

\section{Control glucémico en la población con DMT2} El 46,6\% de los individuos presentó niveles de HbA1c superiores a 7\%. En esta población se presentaron algunas diferencias entre variables sociodemográficas y clínicas; se observó que el riesgo de control 
glucémico inadecuado fue menor en mujeres con respecto a hombres (RP: 0,79; IC 95\%: 0,64$0,96 ; \mathrm{p}=0,021)$. En relación a la presencia de hiperglicemia en ayunas, hubo 3,17 veces más riesgo de generar control glucémico inadecuado (IC 95\%: 2,33-4,32, p<0,05). En la Tabla 2 se muestran las características analizadas según control glucémico.

\section{Síndrome metabólico en la población con DMT2}

EL 64,5\% de los individuos tenía SM, en el análisis bivariado, no hubo asociación entre características sociodemográficas y el síndrome metabólico (p>0,05). En cuanto a las comorbilidades, los individuos con obesidad tuvieron 1,22 veces más riesgo de desarrollar SM (IC 95\%:1,07-1,40; $\mathrm{p}=0,003$ ), y aquellos con hipotiroidismo tuvieron 1,21 veces más riesgo de desarrollar SM (IC 95\%:1,05-1,40; $P=0,009$ ). En lo relacionado a las complicaciones de la diabetes, el riesgo de SM en los individuos con cardiopatía fue más alto, siendo 1,23 más que aquellos sin antecedentes de enfermedad cardiaca (IC 95\%: 1,02 a 1,48; p=0,033). En la Tabla 2 se pueden ver la descripción de las variables y las RP crudas alcanzadas en relación con la condición de SM.

Tabla 2. Características sociodemográficas y clínicas según SM de personas con DMT2

\begin{tabular}{|c|c|c|c|c|c|}
\hline \multirow[b]{2}{*}{ Variable } & \multirow[b]{2}{*}{$\mathbf{n}$} & \multicolumn{2}{|c|}{ Síndrome metabólico a } & \multirow{2}{*}{$\begin{array}{l}\text { RP cruda } \\
\text { IC } 95 \%\end{array}$} & \multirow{2}{*}{ Valor $\mathbf{p}$} \\
\hline & & $\begin{array}{c}\text { No } \\
{[161] 35,5 \%} \\
\end{array}$ & $\begin{array}{c}\mathbf{S i} \\
{[292] 64,5 \%} \\
\end{array}$ & & \\
\hline \multicolumn{6}{|l|}{ Sociodemográficas } \\
\hline \multicolumn{6}{|l|}{ Edad } \\
\hline$<50$ años & 34 & $14\{8,7\}$ & $20\{6,8\}$ & Referencia & 0,505 \\
\hline$\geq 50$ años & 419 & $147\{91,3\}$ & $196\{93,2\}$ & $1,10(0,83-1,47)$ & \\
\hline \multicolumn{6}{|l|}{ Sexo } \\
\hline Hombre & 229 & $84\{52,2\}$ & $145\{49,7\}$ & Referencia & 0,608 \\
\hline Mujer & 224 & $77\{47,8\}$ & $147\{50,3\}$ & $1,04(0,90-1,19)$ & \\
\hline \multicolumn{6}{|l|}{ Zona de residencia } \\
\hline Urbana & 437 & $158\{98,1\}$ & $279\{95,5\}$ & Referencia & 0,054 \\
\hline Rural & 16 & $3\{1,9\}$ & $13\{4,5\}$ & $1,27(1,00-1,63)$ & \\
\hline \multicolumn{6}{|l|}{ Estado Civil a } \\
\hline Con pareja & 244 & $84\{59,6\}$ & $160\{63,2\}$ & Referencia & 0,478 \\
\hline Sin pareja & 150 & $57\{40,4\}$ & $93\{36,8\}$ & $0,95(0,81-1,10)$ & \\
\hline \multicolumn{6}{|l|}{ Clínicas } \\
\hline \multicolumn{6}{|c|}{ Control glucémico (HbA1c) } \\
\hline$\leq 7 \%$ & 242 & $89\{55,3\}$ & $153\{52,4\}$ & Referencia & \\
\hline$>7 \%$ & 211 & $72\{44,7\}$ & $139\{47,6\}$ & $1,04(0,91-1,19)$ & 0,555 \\
\hline \multicolumn{6}{|l|}{ Glicemia en ayunas $^{a}$} \\
\hline 70 a $120 \mathrm{mg} / \mathrm{dl}$ & 175 & $70\{43,8\}$ & $139\{36,1\}$ & Referencia & 0,119 \\
\hline$>130 \mathrm{mg} / \mathrm{dl}$ & 276 & $90\{56,2\}$ & $147\{63,9\}$ & $1,12(0,97-1,30)$ & \\
\hline \multicolumn{6}{|l|}{ Obesidad (IMC $\left.\geq 30 \mathrm{Kg} / \mathrm{m}^{2}\right)$} \\
\hline No & 287 & $116\{72,0\}$ & $171\{58,6\}$ & Referencia & 0,003 \\
\hline $\mathrm{Si}$ & 166 & $45\{28,0\}$ & $121\{41,4\}$ & $1,22(1,07-1,40)$ & \\
\hline \multicolumn{6}{|l|}{ ERC } \\
\hline Estadios I a III & 443 & $157\{97,5\}$ & $286\{97,9\}$ & Referencia & 0,779 \\
\hline Estadios IV y V & 10 & $4\{2,5\}$ & $6\{2,1\}$ & $0,93(0,56-1,55)$ & \\
\hline \multicolumn{6}{|l|}{ Hiperuricemia } \\
\hline No & 436 & $156\{96,9\}$ & $280\{95,9\}$ & Referencia & 0,556 \\
\hline $\mathrm{Si}$ & 17 & $5\{3,1\}$ & $12\{4,1\}$ & $1,10(0,80-1,51)$ & \\
\hline \multicolumn{6}{|l|}{ Hipotiroidismo } \\
\hline No & 358 & $137\{85,1\}$ & $221\{75,7\}$ & Referencia & 0,009 \\
\hline $\mathrm{Si}$ & 95 & $24\{14,9\}$ & $71\{24,3\}$ & $1,21(1,05-1,40)$ & \\
\hline \multicolumn{6}{|l|}{ Enfermedad cardiaca } \\
\hline No & 417 & $153\{95,0\}$ & $264\{90,4\}$ & Referencia & 0,033 \\
\hline $\mathrm{Si}$ & 36 & $8\{5,0\}$ & $28\{9,6\}$ & $1,23(1,02-1,48)$ & \\
\hline \multicolumn{6}{|l|}{ Nefropatía } \\
\hline No & 421 & $148\{91,9\}$ & $273\{93,5\}$ & Referencia & 0,558 \\
\hline $\mathrm{Si}$ & 32 & $13\{8,1\}$ & $19\{6,5\}$ & $0,92(0,68-1,23)$ & \\
\hline
\end{tabular}




\section{Análisis multivariado}

En la Tabla 3 y Tabla 4 se muestran los resultados del análisis de la regresión logística binomial, en el que se presentan las RP ajustadas para las variables independiente control glucémico y SM. Referente a control glucémico, aquellos individuos con hiperglicemia tuvieron 3,12 veces más riesgo de tener HbA1c $>7$ (IC 95\%: 2,28-4,25, p<0,05); los resultados fueron ajustados por edad, sexo y cifras de tensión arterial.
Con relación a la presencia de $\mathrm{SM}$, se obtuvo como resultado que los individuos que habitaban la zona rural tenían 1,48 veces más riesgo (IC 95\%: 1,30-1,56) de desarrollar SM $(\mathrm{p}<0,001)$; este dato está ajustado por edad, sexo, zona de residencia, obesidad, hipotiroidismo y enfermedad cardiaca. También hubo hallazgos estadísticamente significativos ante la presencia de obesidad, hipotiroidismo y enfermedad cardiaca para el desarrollo de SM.

Tabla 3. Modelo multivariado según control glucémico de las personas con DMT2 pertenecientes a un programa de RCV

\begin{tabular}{|c|c|c|c|}
\hline Variable & RP ajustada ${ }^{a}$ & IC 95\% & Valor p \\
\hline $\begin{array}{l}\text { Sexo } \\
\text { Hombre } \\
\text { Mujer }\end{array}$ & $\begin{array}{c}\text { Referencia } \\
0,84\end{array}$ & $0,70-0,99$ & 0,048 \\
\hline $\begin{array}{l}\text { Edad } \\
<50 \text { años } \\
\geq 50 \text { años }\end{array}$ & $\begin{array}{c}\text { Referencia } \\
1,06\end{array}$ & $0,75-1,49$ & 0,733 \\
\hline $\begin{array}{l}\text { Cifras de tensión arterial en consulta } \\
<130 / 85 \mathrm{mmHg} \\
\geq 130 / 85 \mathrm{mmHg} \\
\end{array}$ & $\begin{array}{c}\text { Referencia } \\
0,90 \\
\end{array}$ & $0,66-1,21$ & 0,485 \\
\hline $\begin{array}{l}\text { Glicemia en ayunas } \\
70 \mathrm{a} 120 \mathrm{mg} / \mathrm{dl} \\
>120 \mathrm{mg} / \mathrm{dl}\end{array}$ & $\begin{array}{c}\text { Referencia } \\
3,12\end{array}$ & $2,29-4,25$ & 0,0001 \\
\hline
\end{tabular}

Tabla 4. Modelo multivariado según condición de síndrome metabólico de las personas con DMT2 pertenecientes a un programa de RCV

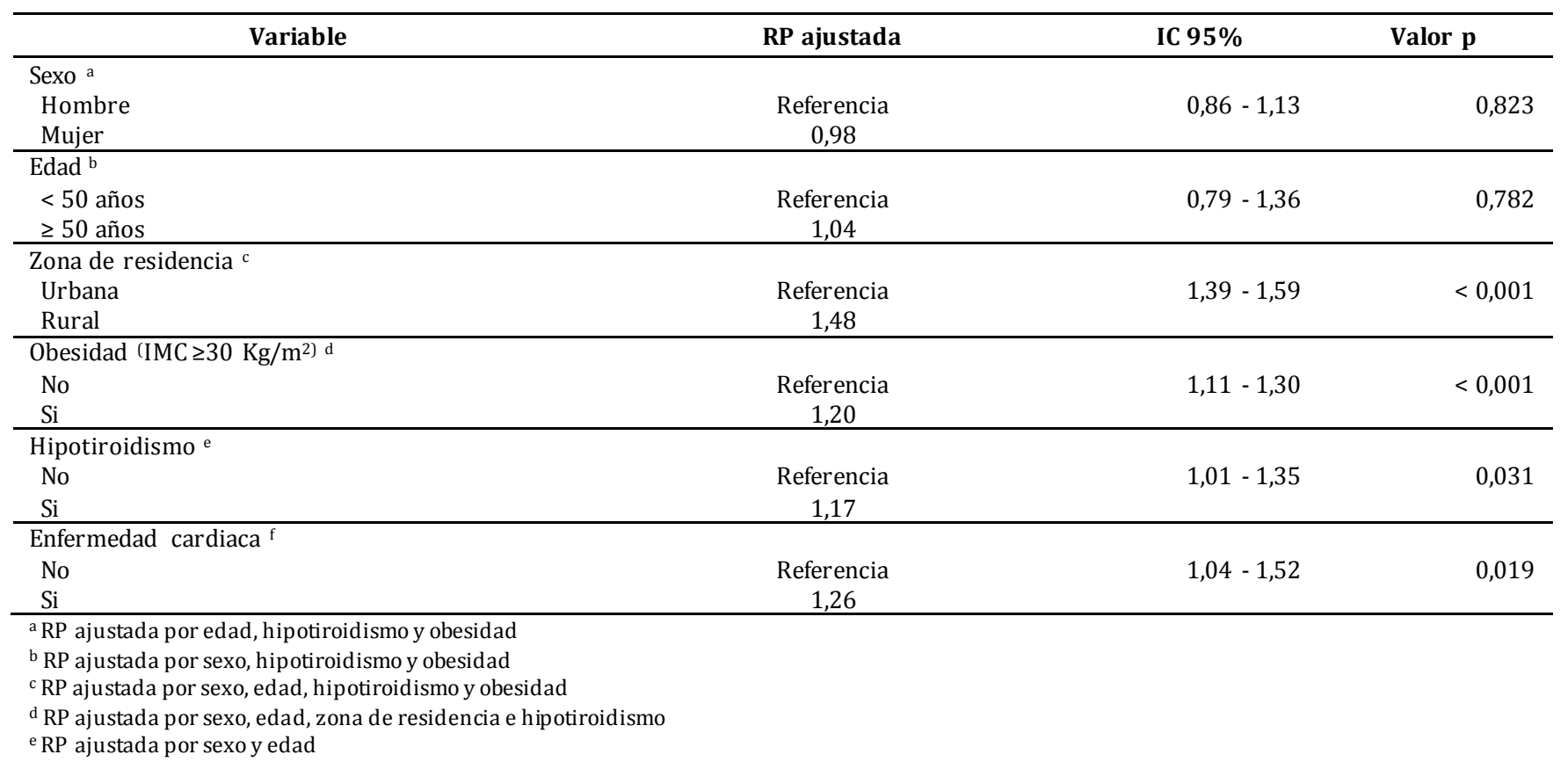




\section{Discusión}

En este estudio se buscó identificar algunos factores clínicos y sociodemográficos potencialmente asociados a una población con DMT2 perteneciente a un Programa Cardiovascular de la capital del departamento del Meta, con el fin de analizar el comportamiento de las variables que rutinariamente son valoradas en los individuos que se encuentran en seguimiento periódico; se monitoreo con dos variables dependientes síndrome metabólico y control glucémico.

Respecto a las características sociodemográficas estudiadas, predominó el sexo masculino $(50,6 \%)$, diferente a otros estudios, donde para este evento, predomina el sexo femenino(17,18), teniendo éste una mayor susceptibilidad a factores genético-ambientales y de otra naturaleza(19). En relación al estado civil, al igual que otros estudios, desarrollados en México sobre cohesión y adaptabilidad familiar y su relación con la HbA1c de los pacientes con DM(20), y sobre necesidades en salud del diabético atendido en el primer nivel de atención(21), mostraron que no existe asociación entre el estado civil y el adecuado manejo de la glicemia en la población diabética. Aunque, en otros estudios se encontró que los individuos con DMT2 con pareja sentimental recibían apoyo social casi tres veces más, que aquellos sin pareja, influyendo positivamente en el manejo de su glicemia(22); por lo cual es necesario profundizar más en el tema.

En relación a la zona de residencia, este estudio mostró que los individuos que habitaban en zonas rurales tenían 1,42 veces más riesgo de desarrollar SM; estos hallazgos fueron diferentes a los encontrados en un estudio desarrollado en España(23) el cual no evidenció diferencias significativas en la prevalencia de la SM entre las dos áreas. Por otra parte, una investigación desarrollada en la región Andina de Colombia, reportó que no hay diferencias importantes en la prevalencia de SM entre las mujeres de ambas zonas de residencia, por el contrario los hombres de la zona rural tuvieron una prevalencia cuatro veces más baja que aquellos que residían en la zona urbana(24).

En relación a las características clínicas, al desconocer el tiempo de diagnóstico y evolución de la DMT2 y sus complicaciones, se estableció como parámetro de control glucémico valores de HbA1c menores o iguales a 7\%, no pudiendo controlar la subestimación de pacientes con corto tiempo de desarrollo de la patología o la sobreestimación en pacientes con daño renal o presencia de anemia(9-11). A pesar de esto, la HbA1c en la actualidad, es el mejor parámetro para valorar la calidad del control metabólico de la población diabética, sobre todo en pacientes que manejan glicemias en ayunas con valores $<180 \mathrm{mg} / \mathrm{dl}$, debido a que por sí sola, la glicemia en ayunas no revela el verdadero estado del control glicémico, por lo que el monitoreo de HbA1c debe garantizarse en todas las áreas de salud cada tres meses $(6,25,26)$.

Por otra parte, este estudio mostró que los individuos con obesidad tenían 1,2 veces más riesgo de desarrollar SM que aquellos con IMC inferior a $30 \mathrm{~kg} / \mathrm{m}^{2}$; la literatura evidencia que estas dos condiciones en personas con DMT2 están interrelacionadas, dado que comparten mecanismos de aparición y evolución, ocasionando complicaciones cardiovasculares(27), pero es importante tener en cuenta que este estimador de obesidad global no es un indicador específico de acumulación de grasa en la región abdominal que ha sido considerado un parámetro importante para SM, puesto que la obesidad central o visceral se determina con circunferencia de la cintura (CC) o los índices de cintura-cadera (ICC) o cintura-talla (ICT) ${ }^{(28,29) .}$

Con respecto a la presencia de ERC, a pesar de que la Guía de Práctica Clínica para el diagnóstico, tratamiento y seguimiento de la DMT2 en la población mayor de 18 años(11), establece que la nefropatía diabética se diagnostica cuando, además de la presencia de microalbuminuria, la TFG está por debajo de 60 $\mathrm{ml} / \mathrm{min}$, calculada utilizando fórmulas como MDRD (Modificación de Dieta en la Enfermedad Renal), Cockroft o, CKD-Epi (Grupo de Colaboración Epidemiológica de la Enfermedad 
Renal Crónica). Se ha evidenciado en los adultos mayores que la TFG estimada por la formula Cockcroft-gault, no es el mejor indicador de función renal en esta población, debido que a partir de los 60 años ocurre una disminución progresiva en la excreción urinaria de creatinina, producto del descenso en la masa muscular asociada al envejecimiento.

La ecuación CKD-Epi usando también métodos de creatinina estandarizados, proporciona ventajas adicionales respecto al MDRD, dado que presenta una mayor exactitud y mejora la capacidad predictiva del filtrado glomerular (especialmente entre valores de 60 y 90 $\mathrm{ml} / \mathrm{min} / 1,73 \mathrm{~m} 2$ ), así como la predicción de mortalidad global y cardiovascular o del riesgo de presentar ERC terminal, por lo cual se considera que CKD-Epi debería sustituir las fórmulas anteriores.(10,30) Al igual que en otros estudios, no se tuvieron en cuenta criterios como la microalbuminuria para analizar ERC, que es un marcador renal de daño endotelial $y$ aterosclerosis temprana(31), debido a que se observó sub registro de esta variable en la muestra analizada (similar a lo que ocurrió con el reporte de medición de circunferencia abdominal).

El $64,5 \%$ de los individuos analizados presentaron SM, que según lo muestra la literatura(8,32), no se trata de una simple enfermedad sino de un grupo de problemas de salud causados por la combinación de factores genéticos y factores asociados al estilo de vida, relacionados especialmente con la sobrealimentación y ausencia de actividad física, aumentado notoriamente el riesgo cardiovascular.

En cuanto a las complicaciones, se determinó que para desarrollar SM dada la presencia de enfermedad coronaria fue $1,26(\mathrm{p}=0,019)$; por el tipo de estudio es difícil concluir cuál de las condiciones se presentó inicialmente; algunos estudios como el desarrollado en La HabanaCuba, sobre características de pacientes de 50 años o menos de edad sometidos a intervención coronaria en los años 2006-2015, y un estudio de Vedanthan et al. relacionado con el análisis de perspectiva global sobre el síndrome coronario agudo: una carga para los jóvenes y los pobres, concluyó que un factor de riesgo relacionado con los dos anteriormente expresados es la dislipidemia; los trastornos lipídicos, la obesidad y la resistencia a la insulina son más frecuentes en pacientes con antecedentes familiares de estas enfermedades o con historia de enfermedad coronaria prematura( 33,34$)$.

La comorbilidad más común fue HTA, similar a al estudio de Roessler(35). Aunque según los reportes de cifras tensionales, más del $85 \%$ estaban normo tensos, es importante analizar que esta información se extrajo de una única consulta y no determina control de cifras tensionales en los individuos durante un periodo de tiempo específico. Tanto la DMT2 como la HTA son factores que aumentan el riesgo cardiovascular y cuando coexisten tiene un efecto mayor en el riesgo de complicaciones tanto macro como micro-vasculares. Este estudio también permitió estimar que los sujetos con hipotiroidismo tenían mayor riesgo de desarrollar SM (RP: 1,17); según la literatura, la presencia de hipotiroidismo se asocia significativamente con componentes individuales del síndrome metabólico(36,37).

\section{Alcances y limitaciones del estudio}

Debido al diseño y métodos de recolección utilizados, este es un estudio que no puede establecer relaciones causa-efecto, pero si permite plantear hipótesis entre variables como las expuestas; de la misma forma, los hallazgos están limitados a la información secundaria extraída de tres fuentes, por lo cual se reconocen potenciales errores de medición, pues no se logró verificar medición de las variables, como la técnica de toma de la presión arterial y el peso, para evitar así variaciones observadordependientes. No obstante, este es un riesgo que se corre en cualquier investigación que emplee información secundaria como el presente. A pesar de esto, se suma al cuerpo de evidencia de los potenciales riesgos asociados en población de pacientes diabéticos en seguimiento, requiriéndose estudios similares o de diseños similares que permitan establecer líneas de intervenciones poblacionales. 


\section{Conclusiones}

El presente es un estudio transversal de una muestra de pacientes de una IPS de Villavicencio. A pesar que este grupo de pacientes se controla y monitorea periódicamente la prevalencia de control glucémico inadecuado fue cercana al $47 \%$, siendo mayor en hombres que en mujeres y asociado a la presencia de hiperglicemia en ayunas.

La prevalencia de SM fue superior al 60\%, éste se asoció con la presencia de zona de residencia (rural), obesidad, hipotiroidismo y en individuos con antecedentes de cardiopatías, luego de ajustar adicionalmente por sexo y edad. Lo anterior evidencia, que aunque los pacientes se encuentran en un programa de seguimiento periódico, un buen porcentaje no alcanza los niveles óptimos de control glucémico y la presencia de condiciones como síndrome metabólico aumenta el riesgo cardiovascular(8). Este estudio de corroborarse con otros estudios similares, indica que es necesario mayor rigurosidad y calidad en los procesos de seguimiento y control pacientes con DMT2 en programas ambulatorios como el expuesto.

Declaración de conflicto de interés: Los autores declaramos que no existen conflicto de interés de ningún tipo, ni real o potencial sobre los resultados presentados.

\section{Referencias}

1. Organización Mundial de la Salud. Informe mundial sobre la diabetes. Ginebra: OMS; 2016.88 p.

2. Organización Mundial de la Salud. Informe sobre la situación mundial de las enfermedades no transmisibles 2014. Ginebra: OMS; 2014.16 p.

3. Aschner P. Epidemiología de la diabetes en Colombia. Adv Diabetol. 2010; 26(2):95-100.

4. Organización Mundial de la Salud. Marco mundial de vigilancia integral para la prevención y el control de las ENT. Ginebra: OMS;2013.

5. Asociación Latinoamericana de Diabetes. Guías ALAD sobre el diagnóstico, control y tratamiento de la Diabetes Mellitus Tipo 2 con medicina basada en evidencia. 13. México D.F: Independencia editorial; 2013.1-141.

6. American Diabetes Association. Standards of Medical Care in Diabetes-2017. 2017.40:142
7. Pereira-Despaigne OL, Palay-Despaigne MS, RodríguezCascaret A, Neyra-Barros RM, Chia-Mena M de los A. Hemoglobina glucosilada en pacientes con diabetes mellitus. MEDISAN. 2015;19(4):555-61.

8. Lahsen R. Síndrome metabólico y diabetes. Rev Médica Clínica Las Condes. 2014;25(1):47-52.

9. Rivas-Vázquez D, Miguel-Soca PE, Llorente-Columbié Y, Marrero-Ramírez GM. Comportamiento clínico epidemiológico del síndrome metabólico en pacientes adultos. Rev Cubana de Med Gen Integr. 2015;31(3):259-69.

10. Martínez-Castelao A, Górriz JL, Bover J, Segura-de la Morena J, Cebollada J, Escalada J, et al. Documento de consenso para la detección y manejo de la enfermedad renal crónica. Endocrinología y Nutrición. 2014;61(9):25-43.

11. Ministerio de Salud y Protección Social, Departamento Administrativo de Ciencia, Tecnología e Innovación Colciencias. Guía de práctica clínica para el diagnóstico, tratamiento y seguimiento de la diabetes mellitus tipo 2 en la población mayor de 18 años. 2016.

12. Plan Decenal de Salud Pública 2012-2021. Ministerio de Salud y Protección Social. Colombia. 2011.237 p.

13. Secretaria de Salud Departamental. Análisis de Situación en Salud con el Modelo de los Determinantes Sociales en Salud. Colombia. 2015.

14. Secretaria Local de Salud de Villavicencio. Análisis de Situación de Salud 2015. Colombia. 2015.

15. Análisis de datos y software estadístico | Stata [Internet]. [cited 2017 Nov 22]. Avaliable from: https://www.stata.com/

16. IBM SPSS - IBM Analytics - España [Internet]. 2017 [cited 2017 Nov 22]. Avaliable from: http://www.ibm.com/analytics/es/es/technology/sps s/

17. Ariza E, Camacho N, Londoño E, Niño C, Sequeda C, Solano C, et al. Factores asociados a control metabólico en pacientes diabéticos tipo 2. Rev Científica Salud Uninorte. 2005; (21): 28-40.

18. Valdés Ramos E, Arjona C, C M del. Características clínicas y frecuencia de complicaciones crónicas en personas con diabetes mellitus tipo 2 de diagnóstico reciente. Rev Cubana Med Gen Integr. 2013;29(2):121 31.

19. Domínguez-Alonso E. Desigualdades sociales y diabetes mellitus. Rev Cubana de Endocrinol. 2013;24(2):200213.

20. Sánchez-Reyes A, Pedraza-Avilés AG. Cohesión y adaptabilidad familiar y su relación con la hemoglobina glucosilada de los pacientes diabéticos. Rev Esp MédQuirúrgicas.2011; 16(2): 82-88.

21. Salinas-Martínez AM, Muñoz-Moreno F, León B de, R A, Villarreal-Ríos E, Núñez-Rocha GM, et al. Necesidades en salud del diabético usuario del primer nivel de atención. Salud Pública México. 2001;43(4):324-33.

22. Arredondo-Motes de Oca A, Márquez-Cardoso E, Moreno-Aguilera F, Bazán-Castro M. Influencia del apoyo social en el control del paciente diabético tipo 2 . Rev Esp Méd-Quirúrgicas. 2006;11(3):43-48.

23. Martínez-Larrad M, Fernández-Pérez C, GonzálezSánchez J, López A, Fernández-Álvarez J, Riviriego J, et 
al. Prevalencia del síndrome metabólico (criterios del ATP-III). Estudio de base poblacional en áreas rural y urbana de la provincia de Segovia. Rev Med Clínica. 2005;125(13):481-86.

24. Aschner-Montoya P. Síndrome Metabólico en una población rural y una población urbana de la Región Andina Colombiana. Rev Med. 2007;15(2):154-162.

25. Barquilla-García A, Mediavilla-Bravo J, Comas-Samper J, Seguí-Díaz M, Carramiñana-Barrera M, ZaballosSánchez F. Recomendaciones de la Sociedad Americana de Diabetes para el manejo de la diabetes mellitus. SEMERGEN- Medicina de Familia. 2010; 36(7):386-391.

26. Tamayo D, Camacho S, López P. Caracterización de pacientes con diabetes mellitus tipo 2 atendidos por médicos residentes de medicina familiar en Bogotá, Colombia. Rev Desafíos. 2015;9(2):17-24.

27. Alegría-Ezquerra E, Castellano-Vázquez J, AlegríaBarrero A. Obesidad, síndrome metabólico y diabetes: implicaciones cardiovasculares y actuación terapéutica. Rev Española de Cardiol. 2008; 61(7): 752-764.

28. Moreira A. ¿Qué medida antropométrica de exceso de peso discrimina mejor el riesgo cardiovascular? Rev Med Clín. 2010;134(9):396-8.

29. Cedeño-Morales R, Castellanos-González M, BenetRodríguez M, Mass-Sosa L, Mora-Hernández C, ParadaArias J. Indicadores antropométricos para determinar la obesidad, y sus relaciones con el riesgo cardiometabólico: cifras alarmantes. Rev Finlay. 2015;5(1):12-23

30. Levin A, Stevens P, Bilous R, Coresh J, Francisco A, Jong $P$, et al. Kidney disease: Improving global outcomes (KDIGO) CKD work group. KDIGO 2012 clinical practice guideline for the evaluation and management of chronic kidney disease. Kidney Int Suppl. 2013;3(1):1150.

31. Gimeno-Orna JA, Blasco-Lamarca Y, Campos-Gutierrez B, Molinero-Herguedas E, Lou-Arnal L, García-García B. Riesgo de mortalidad asociado a enfermedad renal crónica en pacientes con diabetes tipo 2 durante un seguimiento de 13 años. Rev Nefrol. 2015;35(5):48792.

32. Lombo B, Satizábal C, Villalobos C, Tique C, Kattah W. Prevalencia del síndrome metabólico en pacientes diabéticos. Acta Médica Colombiana. 2007; 32 (1): 9-15.

33. Martínez-García G, Valdés-Carrazana E, Cruz-Rodríguez L, Cárdenas-Fernández Y, Ravelo-Dopico R, PereraLombillo C. Características de pacientes de 50 años o menos de edad sometidos a intervención coronaria en los años 2006-2015. Rev Cubana de Med Militar. 2016; 45(2):155-164.

34. Vedanthan R, Seligman B, Fuster V. Global perspective on acute coronary syndrome: a burden on the young and poor. Circ Res. 2014;114(12):1959-75.

35. Roessler E. Manejo de la hipertensión arterial en diabetes mellitus. Rev Méd Clín Las Condes. 2016;27(2):204-12.

36. Sarmiento-Teruel Y, Soca M, Enrique P, AlmaguerHerrera A, Niebla G, Alfonso L, et al. Caracterización del síndrome metabólico en mujeres con hipotiroidismo clínico. Rev Arch Méd de Camagüey. 2013;17(1):51-64.
37. López-Rubio M, Tárraga-López P, Rodríguez-Montes J, Frías-López M del C, Solera-Albero J, Bermejo-López P. Hipotiroidismo subclínico y riesgo cardiovascular. Rev Nutri Hosp. 2015;31(5):2095-102. 